DIAGNOSTICS

\title{
Evaluation of ligase chain reaction for the non-cultural detection of rectal and pharyngeal gonorrhoea in men who have sex with men
}

\author{
H Young, K Manavi, A McMillan
}

Sex Transm Infect 2003;79:484-486

See end of article for authors' affiliations

Correspondence to: Dr Hugh Young, Scottish Neisseria gonorrhoeae Reference Laboratory, Directorate of Medical Microbiology, The Royal Infirmary of Édinburgh, 51 Little France Crescent, Edinburgh EHI6 4SA, UK; Hugh.Young@luht.scot. nhs.uk

Accepted for publication 14 April 2003
Objectives: To compare a nucleic acid amplification test (ligase chain reaction) with culture for detecting rectal and pharyngeal gonorrhoea in men who have sex with men (MSM).

Methods: Duplicate rectal and throat swabs from MSM attending a genitourinary medicine clinic were collected for culture on modified New York City medium and detection of gonococcal nucleic acid by the Abbott ligase chain reaction (LCR) utilising probes based on opa 1 gene sequences. LCR positive culture negative specimens were tested by a second LCR utilising probes based on pilin gene sequences. Patients with rectal and/or pharyngeal cultures yielding Gram negative diplococci confirmed as Neisseria gonorrhoeae by biochemical and immunological methods were diagnosed with rectal and/or pharyngeal gonorrhoea. The criteria for diagnosing rectal and pharyngeal infection by LCR included a positive opa LCR with a positive culture from the same site or, in the case of a negative culture, a positive opa LCR and a positive pilin LCR.

Results: Duplicate rectal samples were obtained from 227 MSM. The results of LCR and culture were concordant in 219 samples (96.5\%). The prevalence of rectal gonorrhoea by LCR and culture was $7.0 \%$ $(16 / 227)$ and $4.0 \%$ (9/227), respectively. Duplicate throat samples were obtained from $251 \mathrm{MSM}$. The results of LCR and culture were concordant in $230(91.6 \%)$ cases. The prevalence of pharyngeal gonorrhoea by LCR and culture was $12.7 \%(32 / 251)$ and $6.0 \%(15 / 251)$, respectively. The specificity of LCR was $99.5 \%(210 / 211)$ for rectal and $98.2 \%$ (215/219) for pharyngeal specimens.

Conclusions: The high prevalence and asymptomatic nature of pharyngeal and rectal gonococcal infection suggests that routine screening for infection at these sites by a nucleic acid amplification test method such as LCR should be considered as part of the overall strategy to control gonorrhoea in MSM.
$\mathrm{R}$ ecent data from west European countries show an increase in gonorrhoea in both women and men, including men who have sex with men (MSM). ${ }^{1}$ In some areas the number of cases of rectal gonorrhoea in MSM has doubled. ${ }^{1}$ As most cases of rectal and pharyngeal infection are asymptomatic, patients infected at these sites make a significant contribution to the transmission of gonorrhoea. There is a lack of surveillance data on pharyngeal gonorrhoea. Rectal gonorrhoea is known to act as an independent factor for HIV transmission in MSM. ${ }^{2}$ The prevalence of rectal gonorrhoea in MSM has also been proposed as a marker of high risk behaviour. ${ }^{3}$

The current gold standard test for detection of gonorrhoea is culture of specimens on modified New York City (MNYC) or Thayer-Martin selective media. However, the heavy colonisation of rectal and pharyngeal mucosae with commensal organisms makes culture from these sites less efficient than cultures from genital sites.

Nucleic acid amplification tests (NAATs) that depend on the amplification of nucleic acid sequences of the target microorganism have the potential for very high sensitivity and specificity, which should make them useful in detecting gonococci at anatomical sites that are heavily colonised by other organisms. To date, none of the NAATs has been licensed for detection of rectal or pharyngeal gonorrhoea. However, screening for asymptomatic genital gonococcal and chlamydial infections has been shown to be cost effective using these methods. ${ }^{4}$

There are very limited data, and none from the United Kingdom, on the sensitivity and specificity of NAATs for detecting rectal $^{5}$ and pharyngeal gonorrhoea. ${ }^{5}{ }^{6}$ The present study is a prospective double-blinded investigation to assess the efficacy of the Abbott ligase chain reaction (LCR) for detecting rectal and pharyngeal gonorrhoea in MSM attending a department of genitourinary medicine.

\section{METHODS}

Consecutive MSM attending the clinic of genitourinary medicine for full screening for sexually transmitted infections over an 8 month period were consented to take part in the present study. After full sexual history was obtained and anogenital examination carried out, screening for gonorrhoea, chlamydia, and syphilis was conducted on each of them according to standard procedures.

Screening for gonorrhoea included urethral, rectal, and pharyngeal culture on MNYC medium. Rectal specimens were obtained by introducing a cotton wool swab in the distal $3 \mathrm{~cm}$ of the anal canal and pharyngeal specimens were taken from the tonsillar area and posterior pharyngeal wall. Swabs were immediately rolled over MNYC plates, which were then held at $37^{\circ} \mathrm{C}$ in a carbon dioxide enriched atmosphere for up to 4 hours before transfer to the laboratory. Cultures were examined after 24 and 48 hours of incubation at $37^{\circ} \mathrm{C}$ in a carbon dioxide enriched atmosphere. Suspect colonies were tested by the oxidase test and oxidase positive Gram negative diplococci were confirmed as Neisseria gonorrhoeae by both immunological (Phadebact Monoclonal GC test) and biochemical tests (rapid carbohydrate utilisation test).

A second rectal and pharyngeal swab was collected from each patient using the Abbott LCR sexually transmitted disease swab collection kit. The swabs were placed in LCR transport media and stored at $-20^{\circ} \mathrm{C}$ before processing in 
Table 1 The sensitivity, specificity, positive predictive value (PPV), and negative predictive value (NPV) of culture and LCR for rectal gonorrhoea

\begin{tabular}{lllll}
\hline & Sensitivity & Specificity & PPV & NPV \\
\hline Culture & $56.3 \%(9 / 16)$ & $100.0 \%(211 / 211)$ & $100.0 \%(9 / 9)$ & $96.8 \%(211 / 218)$ \\
LCR & $100.0 \%(16 / 16)$ & $99.5 \%(210 / 211)$ & $94.1 \%(16 / 17)$ & $100.0 \%(211 / 211)$ \\
\hline
\end{tabular}

Prevalence of rectal gonorrhoea $7.0 \%$ (16/227)

batches according to the manufacturer's protocol for LCR testing. The LCR test utilises four probes based on opa 1 gene sequences. When an LCR positive result was not corroborated by a positive culture from the same anatomical site, the specimen was tested by a second LCR reaction utilising probes based on pilin gene sequences. The laboratory scientists conducting these tests were blinded to the results of the previous tests. The positive opa LCR was considered a true positive if it was positive by the pilin LCR and a false positive if it was negative by the pilin LCR.

The true prevalence of infections was based on a "gold standard" that included all specimens with positive culture results; those with a positive opa LCR from an anatomical site that gave a positive culture; and those with confirmed opa LCR results. The $\chi^{2}$ test was used to assess the statistical significance of differences in test performance between culture and LCR.

\section{RESULTS}

\section{Rectal culture versus $\mathbf{L C R}$}

Duplicate rectal samples were obtained from 227 MSM. The results of LCR and culture were concordant in 219 samples $(96.5 \%)$. The performance characteristics of LCR and culture are summarised in table 1 . The eight discrepancies were LCR positive culture negative samples. Seven samples gave positive pilin LCR assays and were considered as true positives; three of these were from patients with a positive culture at another anatomical site. Therefore, in four of 16 patients with rectal gonorrhoea, the gonorrhoea was not detected by culture at any site. One LCR positive sample gave a negative pilin LCR and was considered a false positive LCR according to our defined criteria (the patient in this case had a positive urethral culture). The prevalence of rectal gonorrhoea by LCR and culture was $7.0 \%$ (16/227) and $4.0 \%$ (9/227) respectively. There was no significant difference in the prevalence of rectal gonorrhoea determined by culture and $\operatorname{LCR}\left(\chi^{2}=2.6 ; \mathrm{p}=0.10\right)$.

\section{Pharyngeal culture versus LCR}

Duplicate throat samples were obtained from 251 MSM. The results of LCR and culture were concordant in 230 (91.6\%) cases. The performance characteristics of LCR and culture are summarised in table 2. The 21 discrepancies were all LCR positive culture negative specimens. Seventeen samples gave positive pilin LCR assays and were considered as true positives; 10 of these were from patients with a positive culture at another anatomical site. Therefore, seven of 32 patients with pharyngeal gonorrhoea were not detected by culture at any site. Four LCR positive samples gave a negative pilin LCR result and were considered as false positive LCR results according to our defined criteria (two were known contacts of gonorrhoea; one had recent multiple casual partners; and one had a history of urethral gonorrhoea 5 months earlier).

The prevalence of pharyngeal gonorrhoea by LCR and culture was $12.7 \%(32 / 251)$ and $6.0 \%$ (15/251). The prevalence of pharyngeal gonorrhoea determined by LCR was significantly higher than by culture $\left(\chi^{2}=6.78 ; p=0.005\right)$.

\section{DISCUSSION}

This study shows that the LCR is a very sensitive method for detecting $N$ gonorrhoeae in rectal and pharyngeal samples. The increase relative to culture in the prevalence of rectal $(4.0 \%$ to $7.0 \%)$ and pharyngeal gonorrhoea $(6.0 \%$ to $13.0 \%)$ supports earlier findings on selected populations. In a subgroup of 47 men with urethral gonorrhoea, Stary et al found that LCR increased the prevalence of rectal and pharyngeal gonorrhoea to $13.0 \%$ from $0 \%$ and $4.0 \%$, respectively, for culture. The specificity of LCR for pharyngeal and rectal samples could not be determined from this study.

In 200 MSM who reported performing fellatio during the previous 2 weeks the prevalence of pharyngeal gonorrhoea was $6.0 \%$ by culture and $11.0 \%$ by LCR. ${ }^{6}$ The sensitivity and specificity of LCR were $94.7 \%$ and $97.8 \%$, respectively, in this study compared with $100.0 \%$ and $98.2 \%$ in our study. The high specificity of LCR is supported by in vitro experiments that found it to be the most specific of the NAATs when tested against laboratory cultures of other Neisseria species. ${ }^{7}$

The high prevalence and asymptomatic nature of pharyngeal and rectal gonococcal infection suggests that routine screening for infection at these sites should be considered as part of the overall strategy to control gonorrhoea and reduce HIV transmission in MSM. It has been shown that oropharyngeal gonorrhoea may be the source of urethral ${ }^{8}$ and disseminated disease, ${ }^{9}$ whereas rectal gonorrhoea is an independent risk factor for HIV transmission in MSM. ${ }^{3}$ The marked superiority in sensitivity of LCR combined with high specificity makes it preferable to culture for screening for rectal and pharyngeal gonorrhoea. This is not only in MSM but also in heterosexual patients whenever pharyngeal (both sexes) and rectal cultures in women are taken according to the testing protocols of individual clinics.

Since the completion of this study it has been announced that the Abbott LCR test will be discontinued in June 2003. As it is generally accepted that the sensitivity of all NAATs is superior to culture this does not alter the significance of the findings of this study and the recommendations made above. However, because false positive reactions can occur with NAATs $^{7}$ it is prudent to consider the initial result as a

Table 2 The sensitivity, specificity, positive predictive value (PPV), and negative predictive value (NPV) of culture and LCR for pharyngeal gonorrhoea

\begin{tabular}{lrrrr}
\hline & Sensitivity & \multicolumn{1}{l}{ Specificity } & \multicolumn{1}{l}{ PPV } & \multicolumn{1}{l}{ NPV } \\
\hline Culture & $46.9 \%(15 / 32)$ & $100.0 \%(219 / 219)$ & $100.0 \%(15 / 15)$ & $92.8 \%(219 / 236)$ \\
LCR & $100.0 \%(32 / 32)$ & $98.2 \%(215 / 219)$ & $88.9 \%(32 / 36)$ & $100.0 \%(215 / 215)$ \\
\hline
\end{tabular}

Prevalence of pharyngeal gonorrhoea $12.7 \%(32 / 251)$. 


\section{Key messages}

- Nucleic acid amplification detected 78\% (16/9) more rectal infections in MSM than culture.

- Nucleic acid amplification detected 113\% (32/15) more pharyngeal infections in MSM than culture.

- $25 \%$ of the patients with rectal infection $(4 / 16)$ and $22 \%(7 / 32)$ of those with pharyngeal infection gave negative cultures for all sites tested and would not have been detected without the use of a NAAT.

- NAATs can contribute to the control of gonorrhoea in MSM by detecting cases of rectal and pharyngeal gonorrhoea that are not currently detected by culture.

"presumptive" positive and to confirm the findings with a second NAAT based on a different target gene.

A major disadvantage in using NAATs such as LCR for diagnosing gonorrhoea is the lack of a gonococcal isolate on which to perform antibiotic susceptibility testing. However, as patients with a positive LCR result will have to return to a clinic for treatment this will provide an opportunity to perform culture. Although the gonococcus will not be cultured in every case, nevertheless such a strategy should provide a significant number of isolates on which to assess the prevalence of resistance to the various antibiotics used in treating gonorrhoea.

\section{CONTRIBUTORS}

$\mathrm{HY}$ and AMcM conceived and designed the study; AMcM supervised the clinical aspects of the study and HY supervised the laboratory aspects; KM undertook a literature review; HY and KM performed data analysis and drafted the manuscript; AMcM reviewed the manuscript.

\section{Authors' affiliations}

H Young, Scottish Neisseria gonorrhoeae Reference Laboratory, Lothian University Hospitals NHS Trust, The Royal Infirmary of Edinburgh, 51 Little France Crescent, Edinburgh EHI6 4SA, UK

K Manavi, A McMillan, Department of Genitourinary Medicine, Lothian University Hospitals NHS Trust, Lauriston Building, Lauriston Place,

Edinburgh, UK

\section{REFERENCES}

1 Nicoll A, Hamers FF. Are trends in HIV, gonorrhoea, and syphilis worsening in western Europe? BMJ 2002;324:1324-7.

2 Burn S, Horner PJ. Rectal gonorrhoea as an independent risk factor for HIV infection in homosexual males. Genitourin Med 1995;71:335-6.

3 Riley VC. Resurgent gonorrhoea in homosexual men. Lancet 1991;337:183.

4 Mehta SD, Bishai D, Howell MR, et al. Cost effectiveness of five strategies for gonorrhoea and chlamydia control among female and male emergency department patients. Sex Transm Dis 2002;29:83-91.

5 Stary A, Ching S-F, Teodorowicz L, et al. Comparison of ligase chain reaction and culture for detection of Neisseria gonorrhoeae in genital and extra-genital specimens. J Clin Microbiol 1997;35:239-42.

6 Page-Shafer K, Graves A, Kent C, et al. Increased sensitivity of DNA amplification testing for the detection of pharyngeal gonorrhoea in men who have sex with men. Clin Infect Dis 2002;34:173-6.

7 Palmer HM, Mallinson H, Wood RL, et al. Evaluation of the specificities of five DNA amplification methods for the detection of Neisseria gonorrhoeae. J Clin Microbiol 2003;41:835-7.

8 Lafferty WE, Hughes JP, Handsfield HH. Sexually transmitted diseases in men who have sex with men. Acquisition of gonorrhoea and nongonococcal urethritis by fellatio and implications for STD/HIV prevention. Sex Transm Dis 1997:24:272-8.

9 Weisner PJ, Tronca E, Bonin P, et al. Clinical spectrum of pharyngeal gonococcal infection. N Engl J Med 1973;288:181-5.

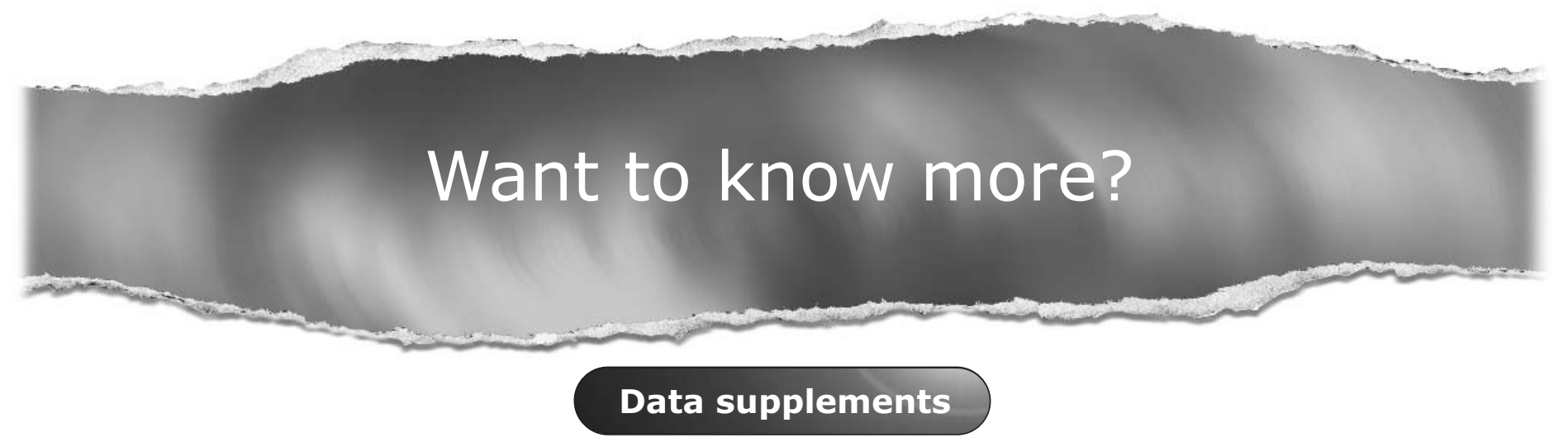

Limited space in printed journals means that interesting data and other material are often edited out of articles; however, limitless cyberspace means that we can include this information online.

Look out for additional tables, references, illustrations.

www.stijournal.com 